\title{
Analytical Modeling of Axial Flux PM Machines with Eccentricities
}

\author{
Yunkai Huang ${ }^{1}$, Baocheng Guo ${ }^{1, *}$, Youguang Guo ${ }^{2}$, Jianguo Zhu ${ }^{2}$, Ahmed Hemeida ${ }^{3}$ and Peter Sergeant ${ }^{3}$ \\ 1 Engineering Research Center for Motion Control of Ministry of Education, Southeast University, Nanjing \\ 210096; gbc_guo@seu.edu.com; huangyk@seu.edu.cn. \\ 2 School of Electrical, Mechanical and Mechatronic Systems, University of Technology Sydney, Sydney, \\ Australia ; Youguang.Guo-1@uts.edu.au; Jianguo.Zhu@uts.edu.au. \\ 3 Department of Electrical Energy, Systems and Automation, Ghent University, Ghent B-9000, Belgium; \\ Ahmed.Hemeida@ugent.be; Peter.Sergeant@ugent.be \\ * Correspondence: gbc_guo@seu.edu.com; Tel.: +86-15005145661
}

\begin{abstract}
In this paper, an analytical quasi three-dimensional method is used to model an axial flux permanent magnet (AFPM) machine with various eccentricities. AFPM machines (AFPMMs) have various advantages but they are sensitive to geometrical imperfections for manufacturing aspect. The main aim of this paper is to propose a general analytical model to analyze the AFPMMs with various types of eccentricities. The radial and tangential magnetic flux densities in the air gap under healthy condition are obtained via combination of Maxwell's equations and Schwarz-Christoffel (SC) mapping firstly. Next, in order to investigate the eccentricities, equations for air gap length and radii are deduced. The back electromotive force (EMF) is calculated and compared with those from healthy condition and finite element (FE) analysis, respectively. The results show that the analytical predictions agree well with the FE results. Moreover, using this method has a significantly less time consuming than the 3D FM simulation process, which is a great advantage of this method. Finally, the analytical model is verified via experimental results.
\end{abstract}

Keywords: Analytical Model; Axial Flux PM Machines; Back EMF; Conformal Mapping; Double Rotor; Eccentricities.

\section{Introduction}

Axial flux permanent magnet machines (AFPMMs) have a number of distinct advantages outstripping radial flux permanent magnet machines (RFPMMs). They can be designed to have a higher power-to-weight ratio resulting in less core material and they are easy to adjust the air gap [1], [2]. Moreover, AFPMMs are smaller in size compared to the RFPMM counterparts, this particular structural characteristic makes them easy to match the space limitation in some applications such as electric vehicles (EV), hybrid electric vehicles (HEV) [3], wind turbines [4] and flywheel storage system [5]. However, due to the smaller contact surface between the rotor and the shaft, AFPMM becomes more difficult to design a rotor-shaft mechanical joint with high mechanical integrity [6]. This makes it predisposed to imperfect assemblies for manufacturing aspect, such as static/dynamic angular eccentricities [7], static/dynamic axis eccentricities [8], and other assembly imperfections which are combined or mixed with these eccentricities [9].

In angular eccentric condition, the rotor is inclined and the air gap is asymmetric. If the rotor shaft assembly is sufficiently rigid, which means that the level of eccentricity does not change during operating period, the axial force becomes unbalanced and this will cause unbalanced magnetic forces (UMFs) and vibrations [10], [11]. UMFs can easily lead to deterioration of both the machine's performance and lifetime. The UMFs can also act on the stator core and expose the stator windings to unnecessary and potentially harmful vibrations, which may even cause the stator and the rotor to scrape [12]. It is therefore important to achieve an estimation of eccentricities by measuring some suitable machine quantities. In [13], five different strategies, temperature measurements, mechanical vibration measurements, chemical measurements, partial discharge detection, and electrical 
measurements are introduced to detect fault conditions in electric machines. Electrical measurements [14], owing to their fast and easy implementation, are widely adopted among electric machine designers. To perform the electrical test, it is very important to model and simulate the electrical machines (EMs) under eccentricities condition in order to predict or diagnose the machine characteristics in such conditions.

Various techniques could be used for modeling AFPMMs under fault conditions, e.g., analytical models, magnetic equivalent circuit (MEC) [15], FE analysis [16] or a combination of these methods [17]. The 3D-FE model can model precisely the influence of eccentricity owing to the accurate computation of air gap region, but it is time consuming because large matrixes need to be solved. Thus, some literatures proposed alternative methods to reduce the computation time. The quasi-3D method, which is widely adopted by AFPMM designers, still has large computation time for eccentricities [18]. Another alternative method is the field reconstruction (FR) method [19], which is a mixed analytical-FE method and partly depends on the FE model. Although it is much faster than 3D-FE model, it still costs much time on modeling the different geometries. Analytical approach, due to its fast and acceptable results, is still regarded as an efficient and favorable method which is widely adopted among EMs designers. From the literature survey, MEC is not accurate enough for some qualities like cogging torque and forces because of node limits. Also, the alignment of rotor and stator makes researchers have to apply several simulation models [20]. In addition, it is more complex when dealing with the non-uniform air gap. Other possible analytical solutions are appearing in the literature [21], [22], but the methods used in both papers have ignored the tangential flux density when modeling the static eccentricity condition.

To overcome the aforementioned problems, this paper adopts an accurate and improved Conformal Mapping (CM) method, Schwarz-Christoffel (SC) method, to investigate the types of eccentricity conditions. $\mathrm{CM}$ method has not been used so far for accurate computation for magnetic field analysis. As it does not depend on FE method, it is not time consuming. In [23], Zarko used this method to calculate the relative permeability of air gap, considering the slotting effect. One of the main assumptions is that the motor has an infinite slot opening, ignoring the shape of slot. Followed by this paper, Zarko improved this method in [24] to handle this problem, but it is a complex approach which needs excellent mathematical foundation. Conversely, SC method can be developed via Matlab SC Toolbox [25] and the assumption of CM model mentioned above could be relaxed to some extent.

This paper is organized as follows. In Section 2, a description of double rotor AFPMM with concentrated coil is provided. Section 3 will introduce the electromagnetic calculations and SC transformations used in this paper. Various eccentricities and their mathematic descriptions will be presented in Section 4. Afterward, the modeling of the healthy and eccentric magnetic distributions is introduced in Section 5. The simulation results, e.g., the flux density in the air gap and back EMFs, will be compared and validated through the simulations done by the FE model. In section 6 , the experimental results verify the accuracy of this method. At the end, conclusions will be presented.

\section{Description of the Prototype AFPM}

Figure 1 shows the construction of the test machine used in this paper. The AFPM machine consists of two rotors and one segmented stator. Soft magnetic composite (SMC) is used for the stator core and axially magnetized NdFeB PMs are mounted on the surface of each rotor, which is composed of \#45 steel. SMC is a relatively new magnetic material and can be an excellent candidate for electrical machine cores with 3D flux path [26], [27]. Three phase windings are concentrated on each stator segment to avoid overlapping. This machine topology benefits from many advantages, including short end windings with high winding fill factor, small stator iron loss at high-speed operation and convenient for manufacturing and assembly, reduced stator weight due to the absence of the stator yoke. Dimensions and specifications of the investigated machine are shown in Table I.

With regards to AFPMM, 3D FE models are more accurate but require much more time than the 2-D multi-slice models. In this paper, Quasi-3D-method is adopted to convert the 3D to 2D model in 
order to reduce the computational time. The machine is divided into certain number of layers. Thus, an axial machine could be considered to be composed of several individual machines, and computations for each plane are done separately. One 2D model developed in the 2D Cartesian coordinates is shown in Fig.1, the average radius of a particular $i$-th layer is given by:

$$
R_{\text {ave }, i}=R_{i}+\frac{R_{o}-R_{i}}{2 n_{s}}(2 i-1)
$$

where $n_{s}$ is the number of slices.

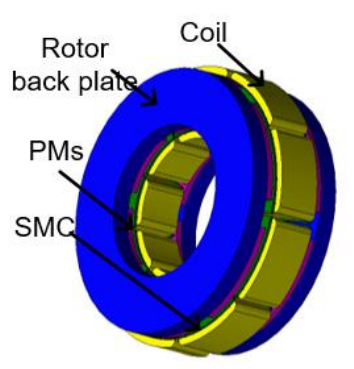

(a) Construction of AFPM

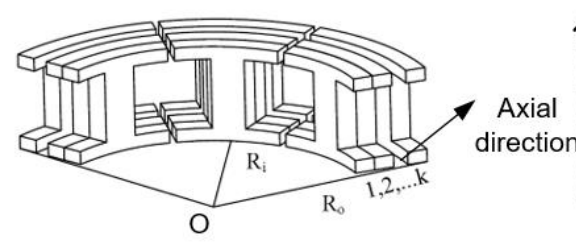

(c) 3D-Stator

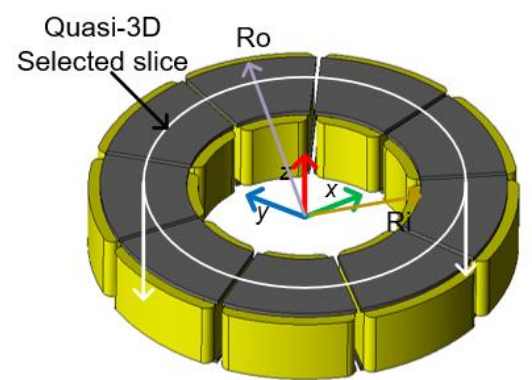

(b) Quasi-3D slice

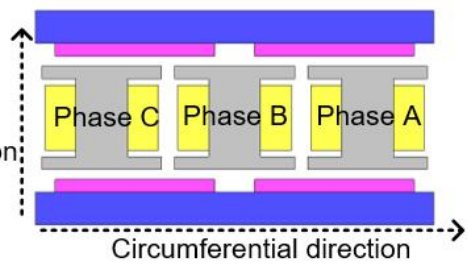

(d) Selected Slice

Figure 1. Construction of the investigated AFPM (a) and the principle of quasi - 3D method (b-d).

Table 1. Parameters of the 6-Pole, 9-Slot AFPM.

\begin{tabular}{cccc}
\hline Parameter & Symbol & Value & Unit \\
\hline Rated power & $P$ & 10 & $\mathrm{~kW}$ \\
Rated voltage & $U$ & 200 & $\mathrm{~V}$ \\
Rated speed & $n_{p}$ & 20000 & $\mathrm{rpm}$ \\
Number of poles / slots & $p / Q_{s}$ & $6 / 9$ & - \\
Stator outer radius & $R_{o}$ & 70 & $\mathrm{~mm}$ \\
Stator inner radius & $R_{i}$ & 45 & $\mathrm{~mm}$ \\
Air gap length & $g$ & 3 & $\mathrm{~mm}$ \\
Remnant flux density of PM & $B_{r}$ & 1.03 & $\mathrm{~T}$ \\
Stator core & - & $\mathrm{SMC}$ & - \\
Permanent magnet & - & $\mathrm{NdFeB}$ & - \\
\hline
\end{tabular}

It should be noted that using mean radius, i.e. using one slice, as a computation plane for modeling may give proper results in AFPMMs as magnet-length to pole-pitch ratio is constant along the machine radius. However, without considering the stator teeth variations and rotor magnet shapes, the results are not accurate enough for computing some machine characteristics which are highly dependent on the shape of teeth and magnets, such as cogging torque, forces, and eddy current loss.

To improve the accuracy of the results, six slices are considered to calculate the healthy and fault conditions in this paper. The processes of quasi method and two poles of a $2 \mathrm{D}$ model in polar coordinates are illustrated in Fig.1 (b-d). 


\section{Calculation of Magnetic Field}

In this paper, the same approach in [20] is used to calculate the magnetic induction in the air gap caused by the magnets and the armature current. Moreover, to take the slot effect into account, the Schwarz-Christoffel mapping [28] is adopted.

By applying Maxwell's equations on selected slice and by summing the air gap flux caused by the armature current and magnets, flux distribution can be obtained. The following assumptions are made during calculations in order to reduce the complexity of the computations.

1. The magnetic material has a uniform magnetization and the relative recoil permeability $\mu_{r}$ is constant and has a value close to unity such as in NdFeB materials.

2. For the computation of armature reaction field, the magnet regions are regarded as free space.

3. Magnetic saturation is absent and the rotor iron cores have infinite magnetic permeability.

4. Eddy current effects are neglected, which avoids the need for the complex eddy current field formulation.

\subsection{Model of PMs}

For $\mathrm{NdFeB}$, the magnetic induction in the PMs is written as:

$$
B=B_{r}+\mu_{r e c} H=\mu_{0} M_{r}+\mu_{r e c} H
$$

where $\mu_{r e c}=\mu_{0} \mu_{r}$ is the recoil permeability.

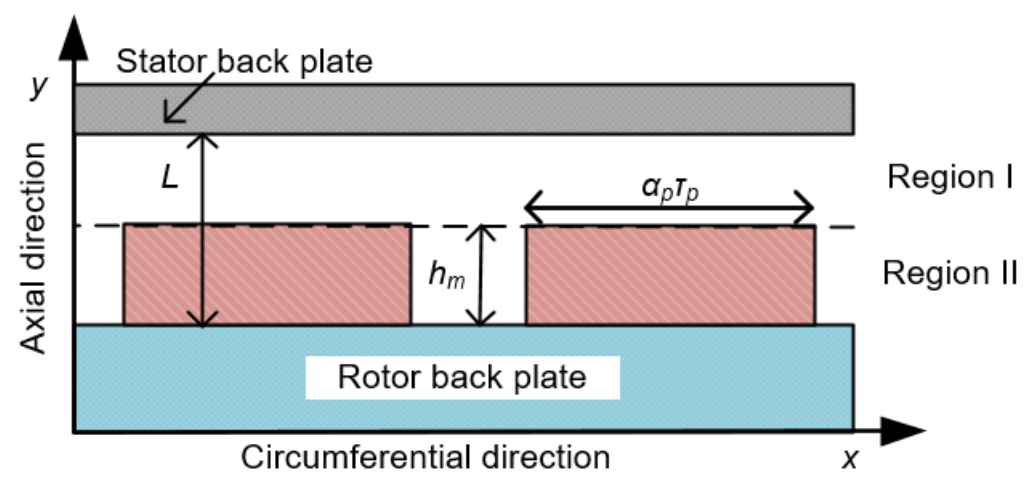

Figure 2. Representation of field regions divided by magnets.

As shown in Fig.2, the magnetization vector is assumed to be along the axial direction and may be described by Fourier series containing only cosine terms.

$$
M_{y}(x)=\sum_{n=1,3,5 \ldots}^{\infty} M_{n} \cos \left(\frac{n \pi x}{\tau_{p}}\right)
$$

where $\tau_{p}$ is pole pitch in circumferential direction and $\alpha_{p}$ is the ratio of magnet pole arc to pole pitch, $h_{m}$ is axial thickness of magnets, $L$ is the axial distance between rotor and back plates and $M_{n}$ is shown as follows:

$$
M_{n}=\frac{4 B_{r}}{n \pi \mu_{0}} \sin \left(\frac{n \pi \alpha_{p}}{2}\right)
$$

For a PM machine with linear demagnetization characteristic, the scale magnetic potentials $\varphi$ in both the air space and the PMs are governed by Laplace's equation when the rectangular coordinate system is adopted.

$$
\frac{\partial^{2} \varphi}{\partial x^{2}}+\frac{\partial^{2} \varphi}{\partial y^{2}}=0
$$

The components of magnetic strength are related to $\varphi$ by 


$$
H_{x}=-\frac{\partial \varphi}{\partial x} \quad H_{y}=-\frac{\partial \varphi}{\partial y}
$$

The following boundary conditions are applied to solution of Eq. (5)

$$
\begin{aligned}
& \left\{\begin{array}{l}
\left.H_{x I}(x, y)\right|_{y=L}=0 \\
\left.H_{x I I}(x, y)\right|_{y=0}=0
\end{array}\right. \\
& \left\{\begin{array}{l}
\left.B_{y I}(x, y)\right|_{y=h_{m}}=\left.B_{y I I}(x, y)\right|_{y=h_{m}} \\
\left.H_{x I}(x, y)\right|_{y=h_{m}}=\left.H_{x I I}(x, y)\right|_{y=h_{m}}
\end{array}\right.
\end{aligned}
$$

By applying the previous boundary conditions, the radial and tangential flux density ( $B_{y}$ and $B_{x}$, respectively) in region I and II are solved. For region I (the air space):

$$
\begin{aligned}
& B_{x I}=\mu_{0} H_{x I}=\mu_{0} M_{n} \sum_{n=1,3,5, \ldots}^{\infty} \frac{\sinh \left(n \pi h_{m} / \tau_{p}\right)}{\Delta} \times \sinh \left\{\frac{n \pi(L-y)}{\tau_{p}}\right\} \sin \left(\frac{n \pi x}{\tau_{p}}\right) \\
& B_{y I}=\mu_{0} H_{y I}=\mu_{0} M_{n} \sum_{n=1,3,5, \ldots}^{\infty} \frac{\sinh \left(n \pi h_{m} / \tau_{p}\right)}{\Delta} \times \cosh \left\{\frac{n \pi(L-y)}{\tau_{p}}\right\} \cos \left(\frac{n \pi x}{\tau_{p}}\right)
\end{aligned}
$$

where

$$
\Delta=\mu_{r} \cosh \left(\frac{n \pi h_{m}}{\tau_{p}}\right) \sin \left\{\frac{n \pi\left(L-h_{m}\right)}{\tau_{p}}\right\}+\cos \left\{\frac{n \pi\left(L-h_{m}\right)}{\tau_{p}}\right\} \sinh \left(\frac{n \pi h_{m}}{\tau_{p}}\right)
$$

\subsection{Model of Stator Slotting}

The effect of stator slotting can be linked to the previous flux density by defining a vector potential $(\lambda)$ in each slot, the radial and tangential components of flux density can be written in the form [23]:

$$
B_{z}=B_{t} \cdot \lambda^{*}=\left(B_{y}+j \cdot B_{x}\right) \cdot(\operatorname{Re}(\lambda)+j \cdot \operatorname{Im}(\lambda))
$$

To this end, the conformal mapping (CM) method is used to calculate the $\lambda^{*}$ by considering the slot effect in electrical machines. The numerical Schware-Christoffel (SC) mapping and its Matlab SC Toolbox could draw the real slot shape.

For radial flux electric machine, the complex relative air gap permeance for slotted air gap can be calculated as [23]:

$$
\begin{gathered}
\lambda=\frac{\partial K}{\partial T} \cdot \frac{\partial T}{\partial Z} \cdot \frac{\partial Z}{\partial S} \cdot \frac{1}{\lambda_{0}} \\
\lambda=\lambda_{y}+j \lambda_{x}
\end{gathered}
$$

where $\lambda_{0}$ is the slotless air gap complex permeance in the T-plane, $\lambda_{x}$ and $\lambda_{y}$ are the tangential and radial components of the complex relative air gap permeance in the original Z-plane. Furthermore, $\mathrm{K}, \mathrm{T}, \mathrm{Z}$ and $\mathrm{S}$ represent the K-plane, T-plane, Z-plane and S-plane, respectively.

It should be noticed that the logarithmic mapping is used for radial flux electric machine to convert the circular geometry in S-plane to Z-plane. However, the AFPM machines in the quasi 3D model [see in Fig.1] can be regarded as linear permanent magnet machines presented by the Z-plane. Hence, the SC mapping only transforms Z-plane to T-plane. However, in order to calculate the 
relative air gap permeance via Hague's solution, the complex K plane is introduced in this paper. In addition, the length of $x$ could be replaced by the equations shown in Fig. 3.

$$
M_{y}(\theta)=\sum_{n=1,3,5}^{\infty} M_{n} \cos \left(\frac{n \pi x}{\tau_{p}}\right)=\sum_{n=1,3,5}^{\infty} M_{n} \cos (n p \theta)
$$

Thus, Eq. (12) can be deduced as:

$$
\lambda=\frac{\partial K}{\partial T} \cdot \frac{\partial T}{\partial Z} \cdot \frac{1}{\lambda_{0}}
$$

After mapping original curved motor geometry to the $\mathrm{Z}$ plane [see in Fig. 3], numerical SC transformation maps one into a polygon with vertices, numbered in counterclockwise order. It should be noted that the crowding phenomenon should be avoided, in which the mapping lines does not distribute in the whole model but only at the corner regions (more detailed information in [18]). The SC transformation in this step maps one canonical domain (e.g. a rectangle, disk, bi-infinite strip, or the upper (lower) half-plane) into the interior (exterior) of the corresponding polygon. This transformation is defined as follows:

$$
z=f(t)=A \int \prod_{k=1}^{n-1}\left(t-t_{k}\right)^{-\frac{\alpha_{t}}{\pi}} d t+C
$$

where $A$ and $C$ are unknown complex constants, $n$ is the number of polygon corners with interior angle $\alpha_{t}, t_{1} \ldots \ldots t_{k}$ are the points in the canonical domain (in the T-plane) corresponding to the polygon corners.

The analytical solution of Eq. (15) is very difficult for geometries with more than three vertices [23], therefore, the SC Toolbox provides a numerical solution by a library of command-line functions which could be seen in [19]. In this paper, the rectangle domain (T-plane) is created by calling $f=$ correctmap (p,alpha), as shown in Fig. 3.

The next step aims to map the interior of annular domain in K-plane to rectangular domain in T-plane. This mapping can be calculated analytically:

$$
t=j\left(\log (k) \frac{\Delta x}{2 \pi}+\frac{\Delta y}{2}-j \frac{\Delta x}{2}\right)
$$

With the determinate rectangular domain vertices by calling $v_{t}=e v a l i n v\left(f, v_{z}\right)$, the length $(\Delta x)$ and width $(\Delta y)$ of the T-plane could be calculated.

Finally, Hague solution for magnetic field in an annular domain could be used, and the final equation which maps the field solution from the K-plane to Z-plane is given as Eq. (14), where $\partial T / \partial Z$ can be obtained in SC Toolbox by calling evaldiff $(f, Z)$ and

$$
\frac{\partial K}{\partial T}=j \frac{\Delta x}{2 \pi} \frac{1}{T}
$$

The air gap field components in T-plane are defined as:

$$
\begin{aligned}
& B_{t-\text { total }}=B_{\mathrm{y}-\text { total }}+j B_{x-\text { total }} \\
& B_{x-\text { total }}=B_{p m x}+B_{\text {armx }} \\
& B_{y \text {-total }}=B_{p m y}+B_{\text {army }}
\end{aligned}
$$

The radial and tangential components of complex relative permeance in the middle of air gap are shown in Fig. 4 (a) and (b), respectively. It shows that, under healthy condition, the complex relative air gap permeance is periodic in each slot pitch. 

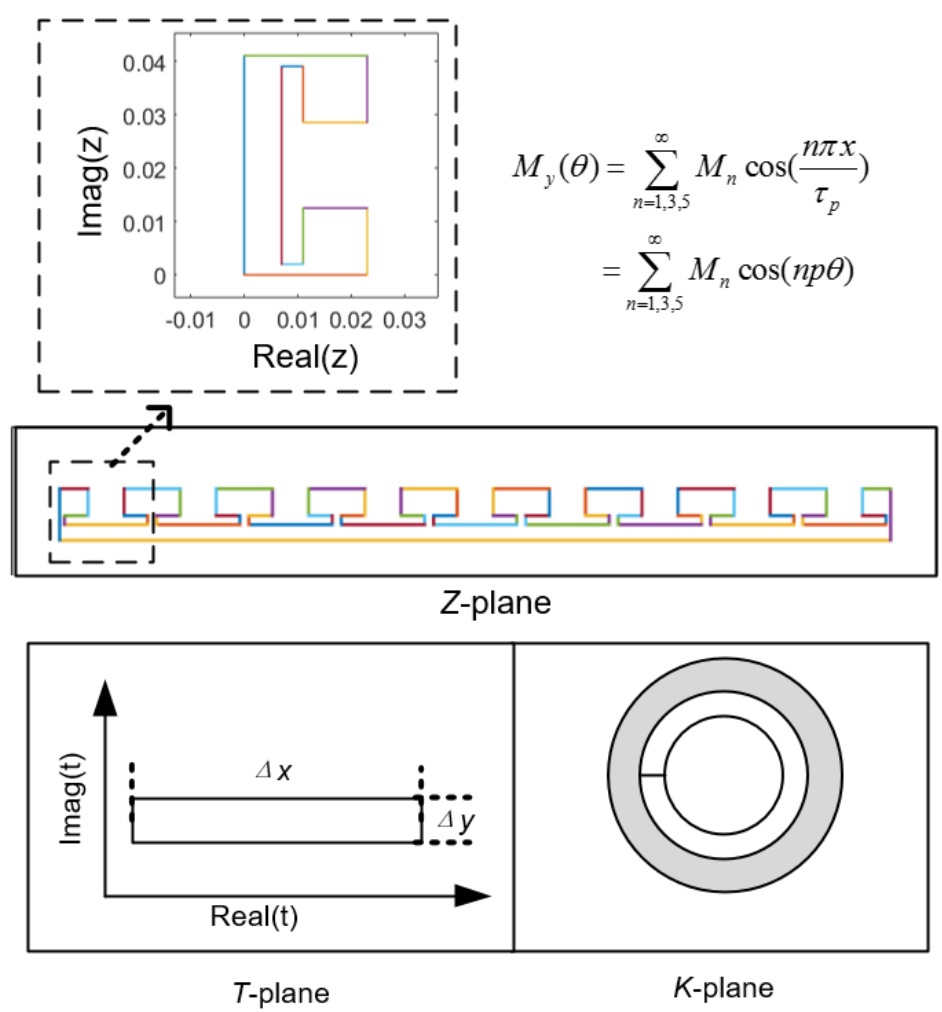

Figure 3. SC mapping in different plane.

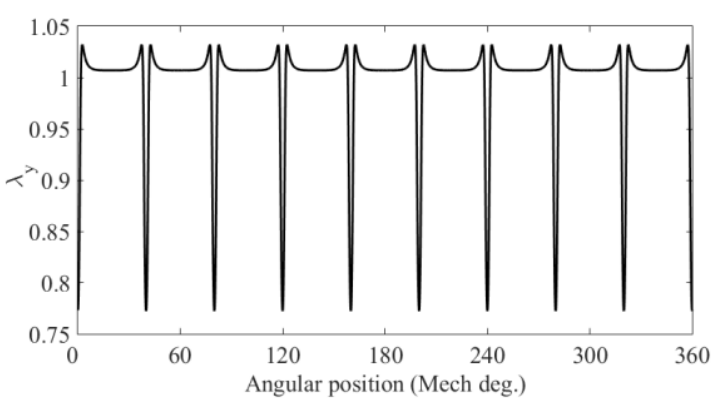

(a)

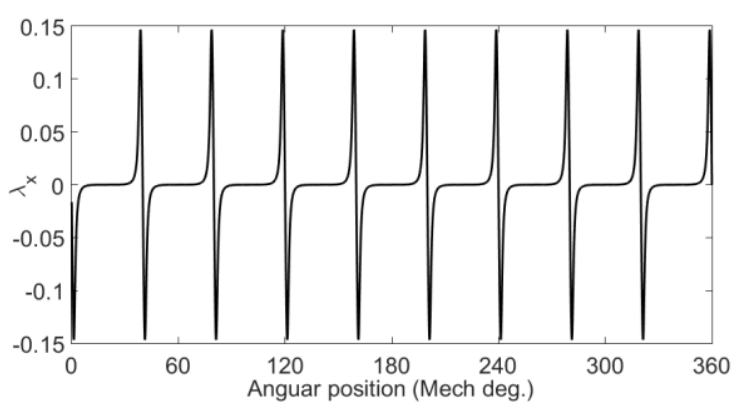

(b)

Figure 4. Complex relative permeance without eccentricity in the middle of air gap. (a) Radial component. (b) Tangential component.

\section{Eccentricities Description}

In this section, four eccentricities, static/dynamic angular eccentricity and static/dynamic axial eccentricity, are presented in detail. Also, the mathematic equations of these eccentricities are introduced.

\subsection{Angular Eccentricity Condition}

Static angular eccentricity is caused by the misalignment of stator and rotor axes but the rotor center is not changed, and thus, the rotor axis is deflected from that of the stator, which causes an ununiform air gap.

In healthy state [see Fig.5 (a)], the air gap length $g_{0}$ is uniform along the circumferential direction. However, in the event of static angular eccentricity occurrence [see Fig. 5 (b)], the rotor shaft experiences a deflection, and the axis of the rotor deviates from that of the stator by an angle $\beta$, and the air gap varies from small to large around the circumference of the stator, and the position does not vary with time. In other words, in the case of static eccentricity the position of the minimum air gap length is fixed in space. 


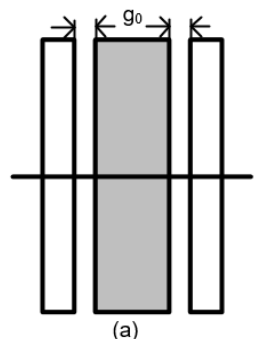

(a)

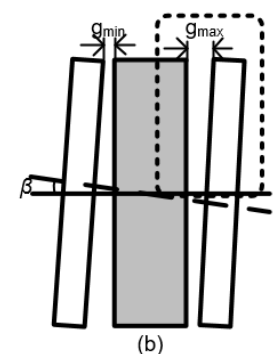

(a)

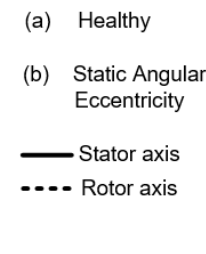

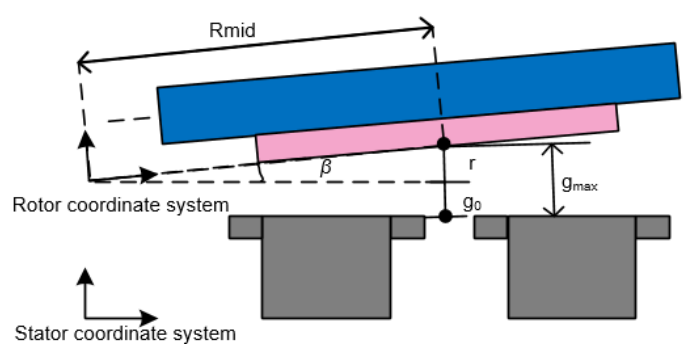

(b)

Figure 5. (a) Double sided topology of AFPM machine in 2D plane; (b) SE in AFPMs

The step in modeling the eccentricity is to evaluate the air gap length in angular eccentricity condition. In [14], The SE factor (SEF) can be defined as:

$$
\mathrm{SEF}=\frac{r}{g_{0}} \times 100 \%
$$

The air-gap length at mean radius $R_{\text {mid }}$ can be written as follows:

$$
g_{m}(\varphi)=g_{0}\left(1-\operatorname{SEF} \cos \left(\varphi-\gamma_{0}\right)\right)
$$

where $\varphi$ is the stator position measured from a reference point $\left(\gamma_{0}\right)$ of the minimal air gap. According to Fig. 5(b), the air gap length in all positions can be understood as:

$$
g(R, \varphi)=g_{m}(\varphi)-g^{\prime}(R, \varphi)
$$

where $g^{\prime}$ is the air gap deviation at the $R_{m i d}$ :

$$
g^{\prime}(R, \varphi)=\left(R-R_{\text {mid }}\right) \sin \beta \cos \left(\varphi-\gamma_{0}\right)
$$

Therefore, the air gap length in all positions can be written as follows:

$$
g(R, \varphi)=g_{0}\left(1-\frac{R}{R_{\text {mid }}} \mathrm{SEF} \cdot \cos \left(\varphi-\gamma_{0}\right)\right)
$$

Because of the high ratio of machine diameter to length in the AFPMMs, $40 \%$ SEF means that the maximum declined distance of rotor plate is $1.2 \mathrm{~mm}$ in this case. SEF of more than $40 \%$ will not be considered in this paper.

In this paper, as quasi-2D model processed in Fig. 1, the minimal air gap happens at $\gamma_{0}=0 \mathrm{rad}$ and the maximum air gap at $\varphi=\pi \mathrm{rad}$.

The dynamic angular eccentricity in the AFPMM is also caused by the declined rotor plane but it can be seen that the rotor axis is not changed from the configuration presented in Fig. 6 .

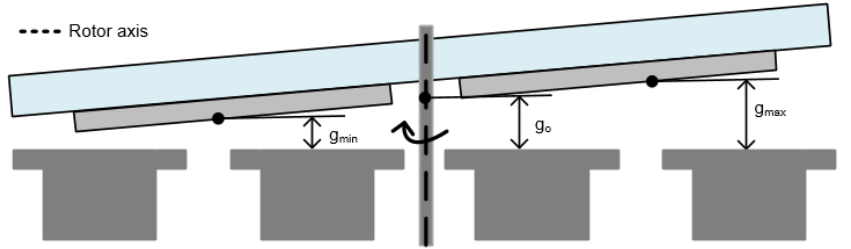

Figure 6. Configuration of dynamic angular eccentricity condition.

For pure dynamic angular eccentricity, each individual pole on the rotor has a constant air gap length for rotating reference frame but a sinusoidal variation (static reference frame) of air gap for each rotor revolution. Thus, the equation of dynamic angular eccentricity can be deduced from Eq. 23 as follows: 


$$
g(R, \varphi)=g_{0}\left(1-\frac{R}{R_{\text {mid }}} \mathrm{SEF} \cdot \cos \left(\varphi-\gamma_{0}-\omega t\right)\right)
$$

where $\omega$ is the angular velocity and $t$ is the time instant.

Fig. 7 shows the magnitude of air gap length in two moments of simulation time under the static and dynamic angular eccentricity.

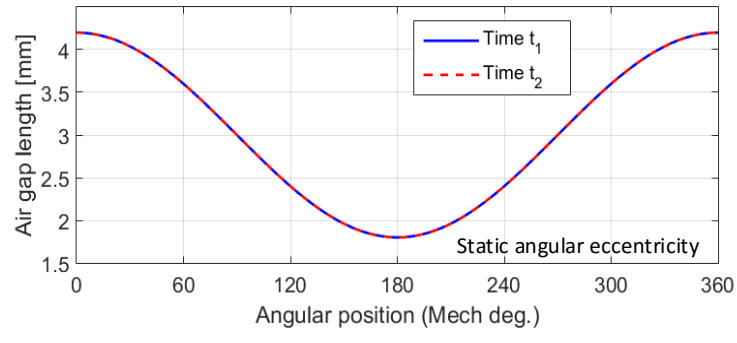

(a)

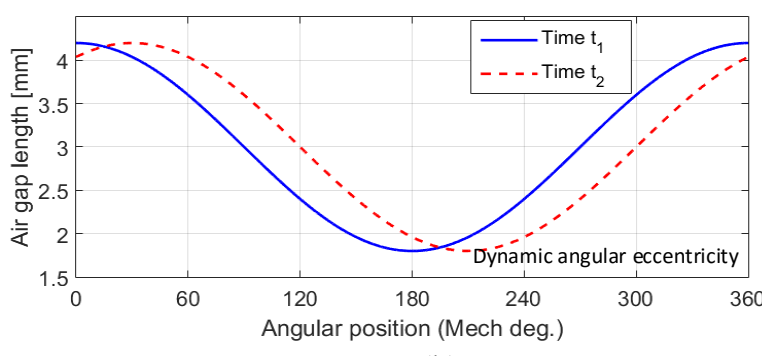

(b)

Figure 7. The change of air gap length under different angular eccentricities. (a) static angular eccentricity condition; (b) dynamic angular eccentricity condition.

\subsection{Axis Eccentricity Condition}

In this paper, the static axial eccentricity occurs when the stator and rotor are offset from one another but the rotor is spinning with its own center, as shown by the representation of Fig. 8 (a).

In [8], Thiele and Heins deduced the radius of eccentricity Reccs, and the time change angle $\beta_{s}$ [see Fig. 8 (b)]. For instance, the middle radius of stator can be mapped to the corresponding radius of rotor, and they can be determined as:

$$
\begin{gathered}
R_{e c c}(\theta)=\sqrt{R_{m i d}^{2}+E_{c c s}^{2}-2 R_{m i d} E_{c c s} \cos \left(\pi-\theta_{s}\right)} \\
\beta_{s}=\operatorname{acos}\left(\frac{R_{m i d}^{2}+R_{c c c}^{2}-E_{c c s}^{2}}{2 R_{\text {mid }} R_{e c c}}\right)
\end{gathered}
$$

where $E_{c c s}$ is the maximum offset distance and $\theta_{s}$ is the location of the slot at angle $\theta$.

Dynamic eccentricity occurs when the rotor shaft is offset from the rotor center, as shown in Fig. 9. Similarly, it has the same characteristics, from a rotating frame, that the pole has a constant eccentricity while the slot in the stator has a sinusoidal variation from the static reference frame.

A similar procedure is used to deduce the dynamic axis eccentricity, and hence the radius of eccentricity can be described as:

$$
R_{e c c}(\theta)=\sqrt{R_{\text {mid }}^{2}+E_{c c s}^{2}-2 R_{\text {mid }} E_{c c s} \cos \left(\pi-\theta_{s}-\omega t\right)}
$$

The magnitude of Recs in two moments of simulation time under static/dynamic axis eccentricity $\left(E_{c c s}=4 \mathrm{~mm}\right)$ are shown in Fig. 10. As shown, the magnitude is unchanged under static eccentricities (for both angular and axial). There is only phase shift under the dynamic eccentricities. 

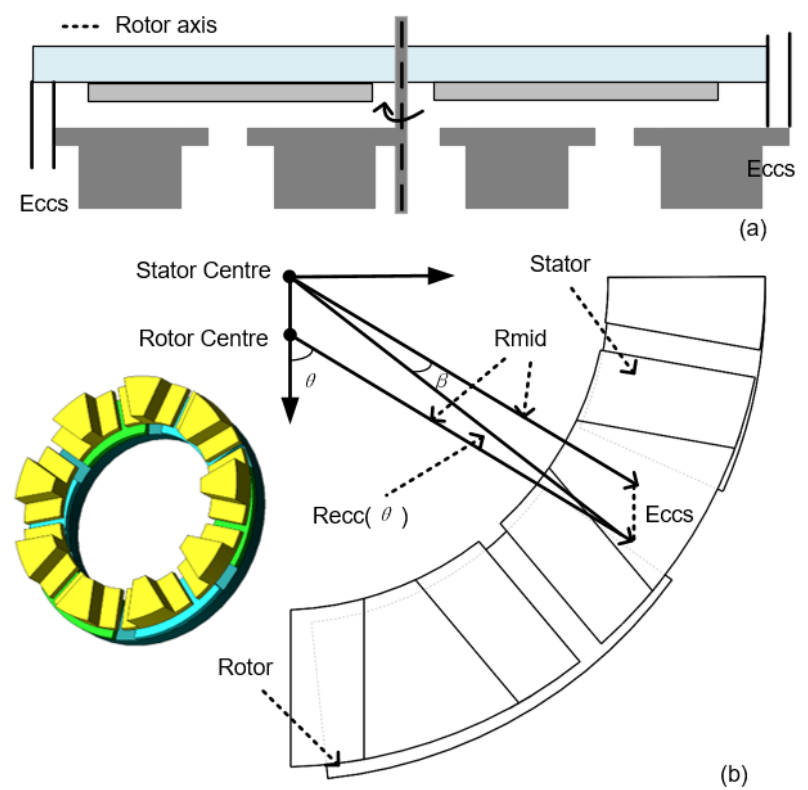

Figure 8. Configuration of static axis eccentricity condition.

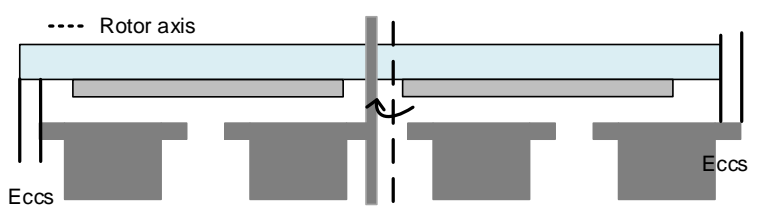

Figure 9. Configuration of dynamic axis eccentricity condition.

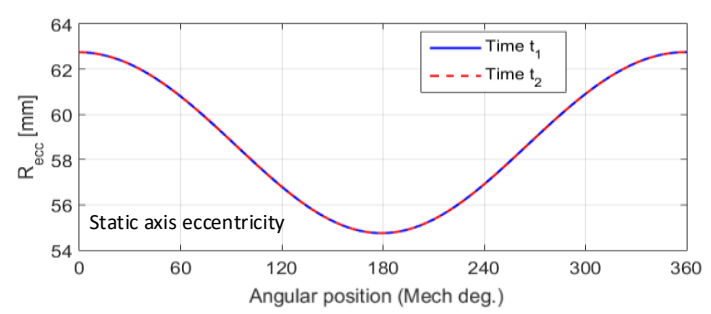

(a)

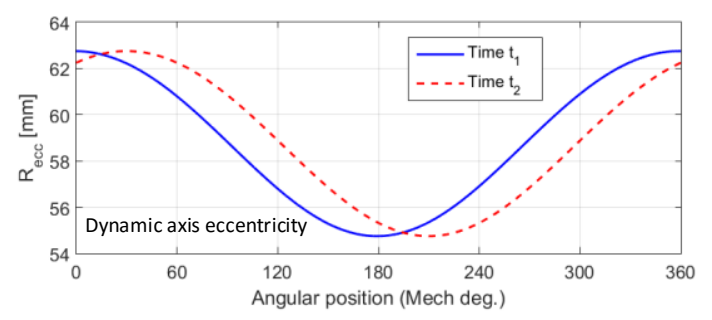

(b)

Figure 10. The length of $R_{e c c}$ under static/dynamic angular eccentricity.

\section{Results and Discussion}

To validate the analytical model, an axial flux permanent magnet machine with 9 slots and 6 poles has been studied. The basic parameters are shown in Table I.

In the analysis, as shown in Fig. 11, the flux density is assumed positive if oriented from left to right. Thus, in the general stator tooth, an incoming flux from the left rotor disk and an outgoing flux towards the right rotor disk are assumed, disregarding the PM orientation, the coils are symmetrical with respect to the teeth axes. This fact determines the symmetry of the tooth field lines between adjacent teeth. Thus, the flux linkage of the $k_{t h}$ tooth coil with $N_{c}$ turns is simply given by:

$$
\psi_{k}=N_{c}\left(\psi_{L k}+\psi_{R k}\right) / 2
$$




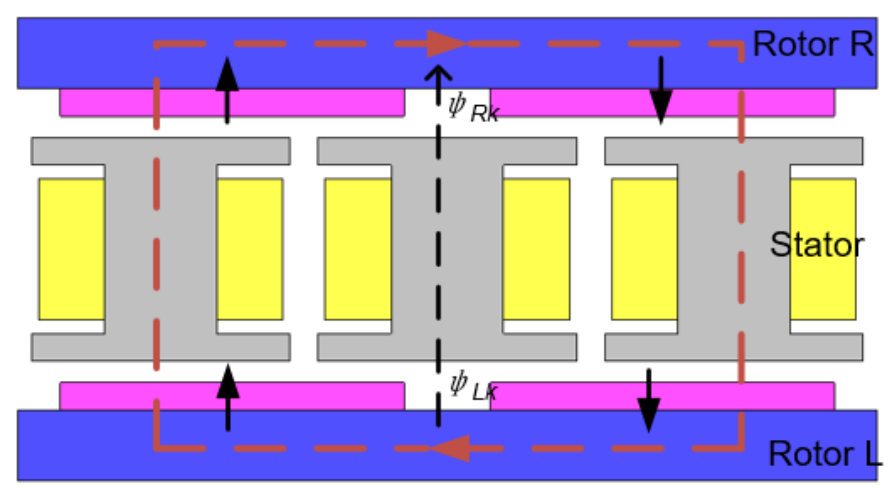

Figure 11. Right and left magnetic flux in the tooth.

The flux linkage is equal to the integral of the air gap flux density distribution across one coil pitch, which is given by:

$$
\psi_{c, i}=\frac{R_{a v e, i}\left(R_{o}-R_{i}\right)}{n_{s}} \int_{\theta_{0}}^{\theta_{0}+\theta_{c}} B_{y, i} d \theta
$$

where $\theta_{0}$ is the coil starting side angle from origin and $\theta_{c}$ is the angle of coil pitch, and $B_{y, i}$ is the axial component of flux density in $i_{\text {th }}$ layer.

The back-EMF per phase is calculated in all coils of the phase winding connected in parallel. In the test machine for phase $A$, according to coils arrangement as shown in Fig. 1, the back-EMF in $i$ th layer $E_{A, j}$ is given by:

$$
E_{A, i}=-N_{c} \frac{R_{a v e, i}\left(R_{o}-R_{i}\right)}{n_{s}} \frac{d \psi_{c, i}}{d t}
$$

And based on the quasi-3-D consumption, the back EMF for the whole machine is calculated as:

$$
E_{A}=\sum_{i}^{N_{i}} E_{A, i}
$$

The cogging torque is another important parameter for PM machines. It occurs in slotted AFPM machine due to interaction between the PM magnetic field and variable permeance of air gap due to stator slots. The cogging torque is independent of armature currents and is created only by the PM magnetic field. The period of the cogging torque is calculated as follows:

$$
\text { Cogging torque period }=2 \pi / \operatorname{LCM}\left(Q_{s}, 2 p\right)
$$

where $\operatorname{LCM}\left(Q_{s}, 2 p\right)$ is the least common multiple of the stator slot number and the rotor pole number.

The cogging torque is calculated by the changing rate of total air gap co-energy including the region of PMs [25]:

$$
T_{\text {cog }}=\frac{\partial W}{\partial \theta}=\frac{\partial}{\partial \theta}\left(\iiint_{\tau} B_{t-\text { total }}^{2} d \tau\right)
$$

Both rotors contribute to the cogging torque, and thus the cogging torque of two disks should be superimposed.

\subsection{Healthy Results}

The radial and tangential components of the magnetic field at two time steps are shown in Fig. 12. The figures show a good agreement between the analytical and FE model. 

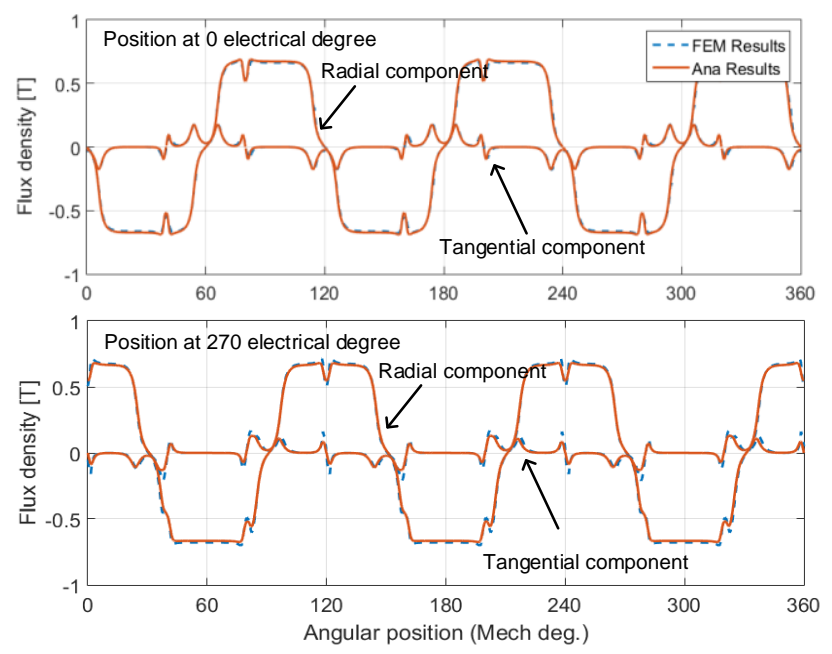

Figure 12. Radial and tangential components of flux density at the center of air gap of the PM at two time steps (120 mechanical degrees per cycle).

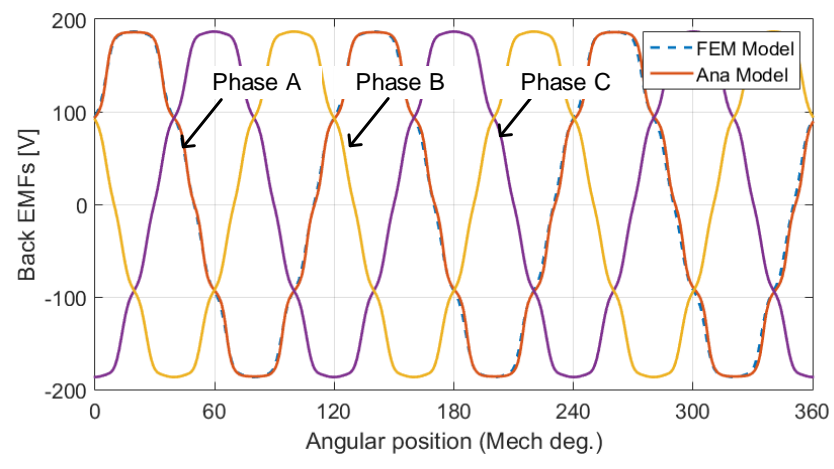

Figure 13. Back EMFs under healthy condition.

In addition, the back EMFs are induced from Eq. 28-31, combined with the magnetic flux density calculated previously. The back EMFs are shown in Fig. 13, and only phase $A$ is compared with the FE model and a good agreement can be seen in the figure.

Fig. 14 shows the cogging torque under healthy condition. It can be seen that the number of cogging ripples is equal to the value calculated by Eq. 33 . The comparison between the FE and analytical models is very good.

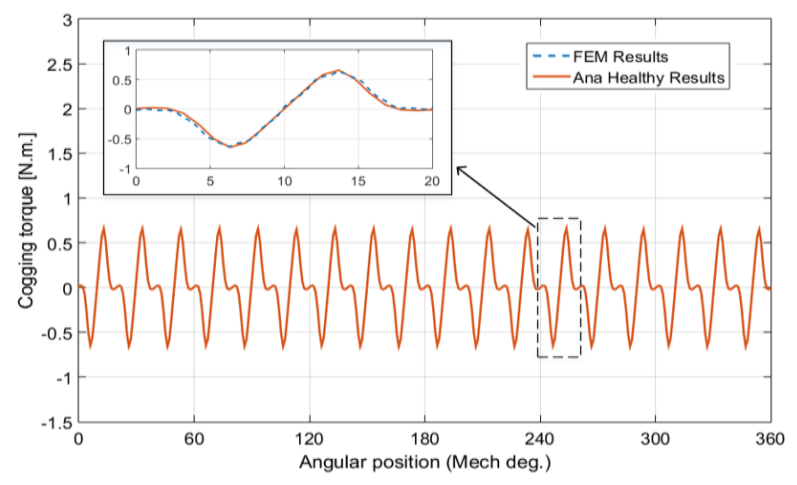

Figure 14. Cogging torque under healthy condition.

\subsection{Angular Eccentricity Results}

Now, in order to simulate the angular eccentricity model, the rotor of AFPM is assumed with $40 \%$ eccentricity, which means that the rotor plane has declined by $1.2 \mathrm{~mm}$. Moreover, it should be 
noted that the FE model of AFPMM should be modeled with two rotor disks to ensure the real flux density distribution.

The radial and tangential components of one side rotor disk at $40 \%$ eccentricity are shown in Fig. 15. It can be seen that, the error of radial and tangential flux density between the FE model and analytical results is increased. This due to the inclined rotor plane which result in strong interaction between the tangential and radial flux densities. Hence, the target of this analytical model is not accurately enough to predict the cogging torque value, but to qualitatively reveal influence of the flux density distribution. The error is in reasonable limit and it can obtain an accurate EMFs calculation.

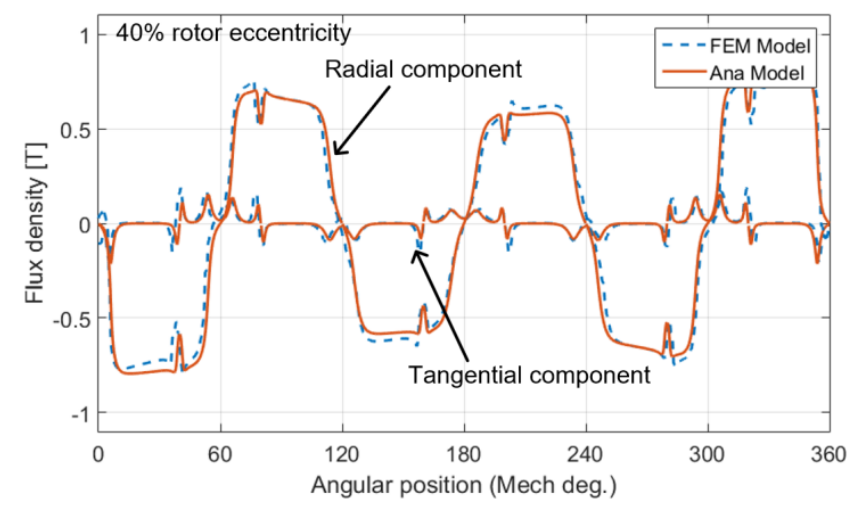

Figure 15. Radial and tangential components of flux density at the air gap ( $l=L-1 \mathrm{~mm})$ under no-load condition (180 mechanical degrees per anti cycle).

Comparing with the healthy condition, it can be seen that the flux density at the near minimum air gap has increased but the flux density at the near maximum air gap has decreased.

In order to illustrate the difference between static and dynamic angular eccentricities, the flux densities at one disk when rotating 180 degrees are presented in Fig. 16. It can be seen that the amplitude of flux density under dynamic angular eccentricity is changing with time but it does not change under static angular eccentricity.

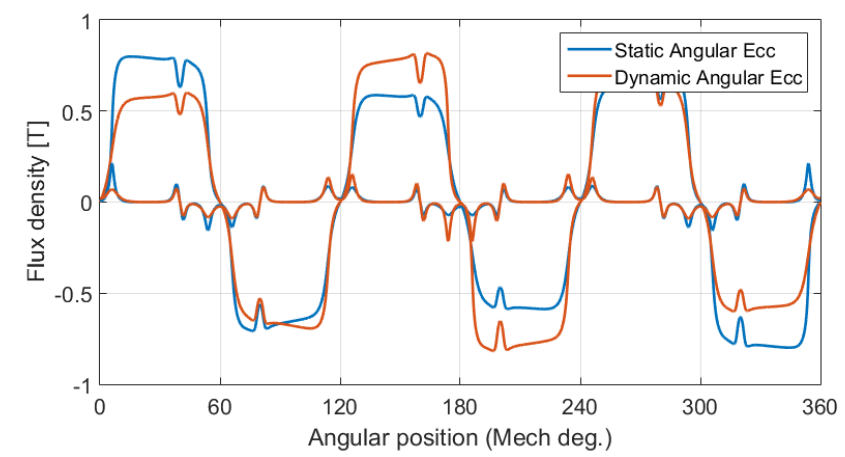

Figure 16. Radial and tangential components of flux density at the air gap $(l=L-1 \mathrm{~mm})$ when rotating 180 degrees (180 mechanical degrees per anti cycle).

The back-EMFs of the test machine under static/dynamic angular eccentricities are compared in Fig. 17.

It can be seen that the back EMF is slightly increased but has the same phase, and from Fig. 17, we can deduce that monitoring the back EMF is not an effective approach to detect angular eccentric faults in double rotor AFPMMs. In healthy condition, all induced voltages of the coils have the same shape and amplitude. However, under angular eccentricity, the back EMF is not affected by eccentricity sufficiently in SE condition. The eccentricity increases the air gap, but on the other hand, the length of air gap of corresponding position is decreased. These two effects seem to compensate each other, given the same results. 


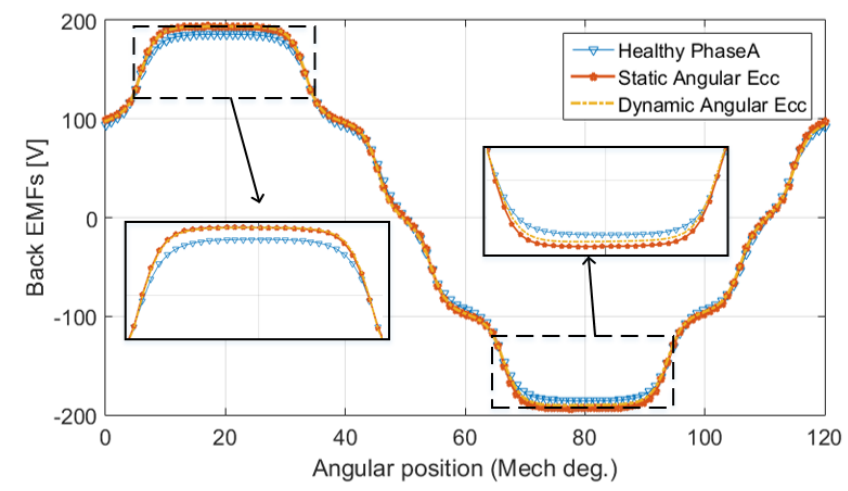

Figure 17. Back EMFs under static/dynamic angular eccentricities.

Compared with radial flux PM machine, the radial and tangential flux density of AFPMM has a strong interaction under angular eccentricity conditions, and hence the analytical model is not suitable for the cogging torque calculation, and hence, the cogging torque will not be discussed in this section.

\subsection{Axis Eccentricity Results}

In order to simulate the axis eccentricities, the parts of poles overhanging the outer and the inner radius of the stator are ignored.

As previous illustration, the maximum axis eccentricity $\left(E_{c c s}\right)$ is set as $4 \mathrm{~mm}$, and the initial angle $(\theta)$ shown in Fig. 8 is set as 0 degree.
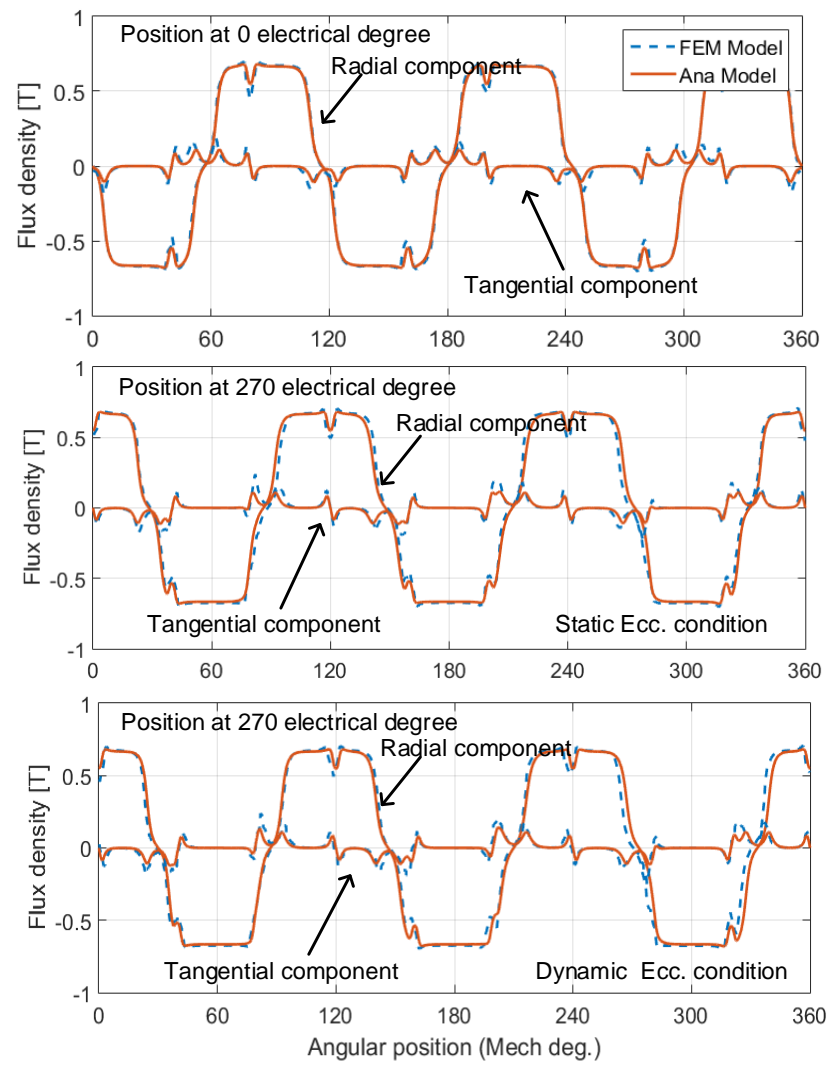

Figure 18. Radial and tangential components of flux density at the air gap $(l=L-1 \mathrm{~mm})$ under static/dynamic axis eccentricities (120 mechanical degrees per cycle).

With regard to the static/dynamic axis eccentricities, the changed radii of eccentricity are induced into (4) and (5) combined with changed angle. From Fig. 8, the axis eccentricities could result in the change of the pole-arc ratio, and hence the period of flux density wave is no longer equal to 
each other (60 mechanical deg. under healthy condition). Fig. 18 presents the flux density under axis eccentricities at two time steps.

The calculated flux densities under eccentricities are in good agreement with the results of FE model, and in order to illustrate the difference of two eccentricity types, the comparison of flux densities when rotor rotating 180 mechanical degrees are also shown in Fig. 19.

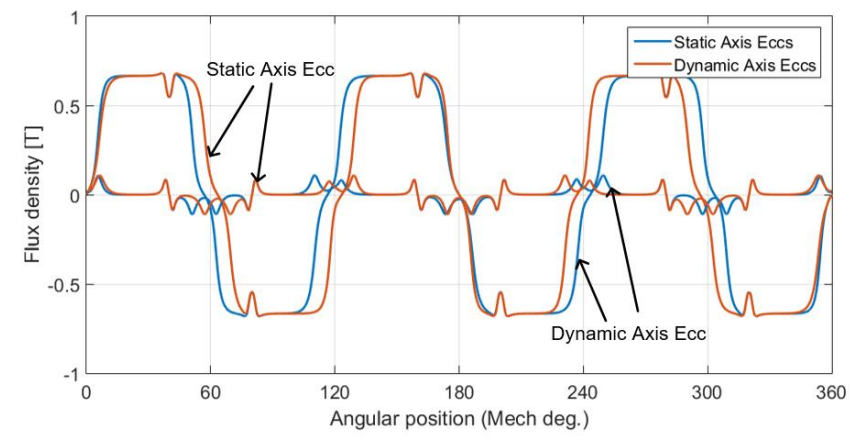

Figure 19. Radial and tangential components of flux density at the air gap $(l=L-1 \mathrm{~mm})$ when rotating 180 degrees (180 mechanical degrees per anti cycle).

From the simulation results and comparison, it can be seen clearly that the bandwidth of flux density is not equal along the circumference direction, and the width of static axis eccentricity does not change with time but the dynamic eccentricity does.

The back EMFs deduced by the static axis eccentricity are shown in Fig. 20. It can be seen that the amplitude of phase $A$ EMF is decreased and the value is equal at each electrical period. This phenomenon can be simply explained by the pole-arc ratio which is decreased at the phase $A$ position. In addition, it does not change with time, which would result in the decrease of integration in Eq. 24.

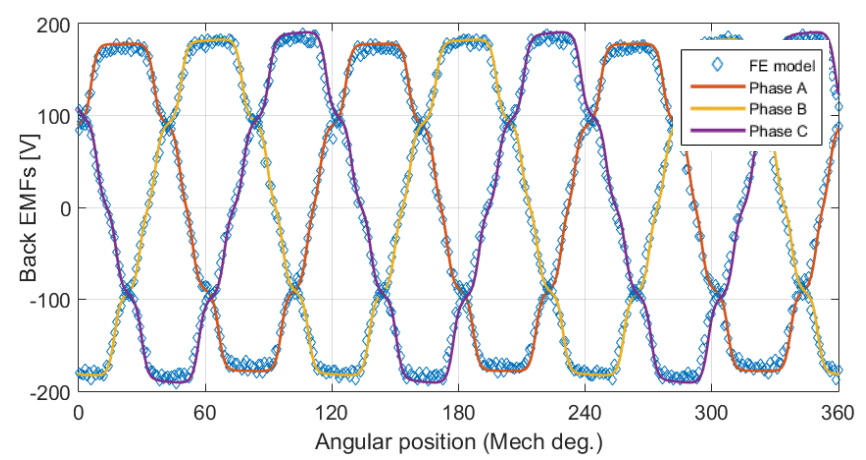

Figure 20. Back EMFs under static axis eccentricity.

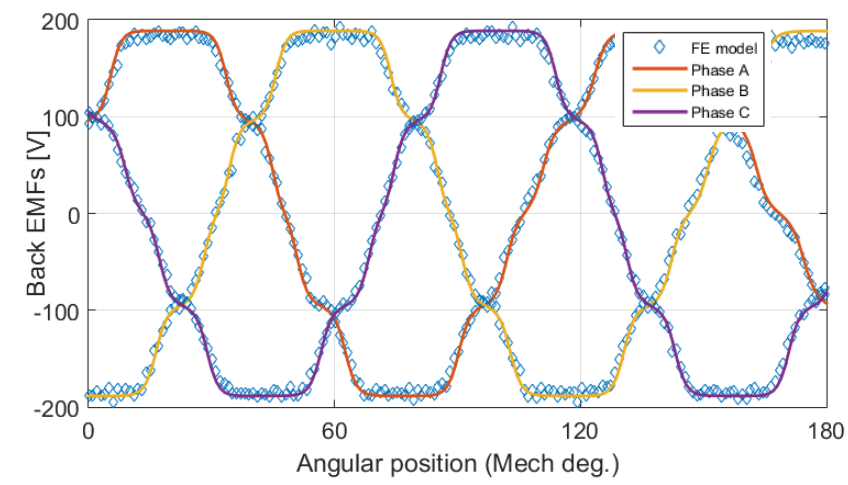

Figure 21. Back EMFs under dynamic axis eccentricity.

For comparison with the back EMFs of static axis eccentricity, Fig. 21 shows that of dynamic axis eccentricity. It can be seen that the proposed method is in good agreement with the FE model. 
In order to illustrate the difference of back EMFs between healthy condition and static/dynamic axis eccentricities, phase $A$ is investigated, as shown in Fig.22. It can be seen clearly that the backEMF of dynamic model has an angle shift and also a slight shift for the static eccentricity. Through detecting the amplitude, the static eccentricity offset position can be easily found but that for dynamic eccentricity cannot.

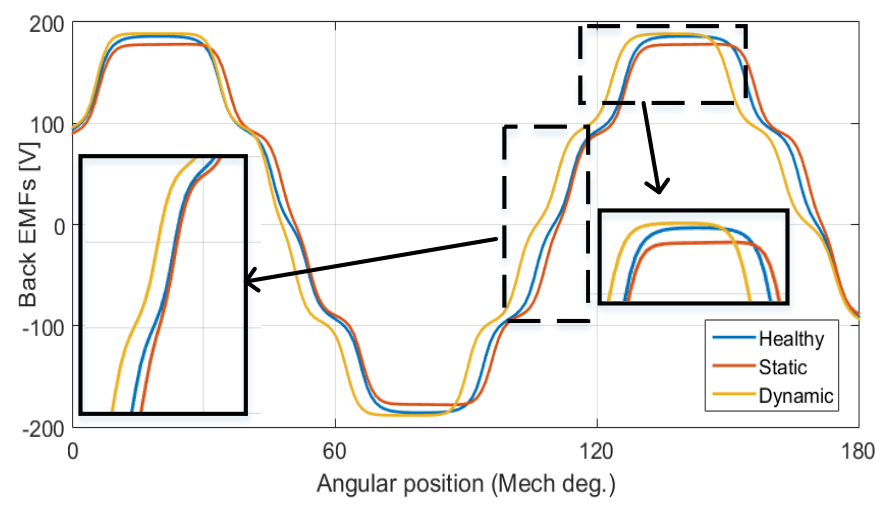

Figure 22. Comparison of back EMFs under axis eccentricities.

\section{Experimental Results}

The double rotor AFPMM in Table I is the prototype investigated in this paper. The rotor disk and the prototype machine are shown in Fig. 23 (a) and (b), respectively. The experimental set up and devices are shown in Fig. 23 (c). The AFPM motor is driven by a frequency converter to test its motor performance, and the no-load parameters, such as the back EMF, is tested by using a driving motor via a belt.

As for the experiment, the no-load test is operated at 300rpm and the back EMF is measured as shown in Fig. 24. It is highly similar with the analytical results of Fig. 13. In other words, the analytical results show a good agreement with the experiment results. From the measurement data, the back EMF of phase $A$ is 118.7 electrical degrees ahead of that of phase $B$ and phase $B$ is 119.2 electrical degrees ahead of that of phase $C$. Table 2 shows the back EMF coefficients obtained by analytical model, FE model and experiment. However, there is a little RMS error between the analytical results and real values. This may be caused by the belt-driven system.
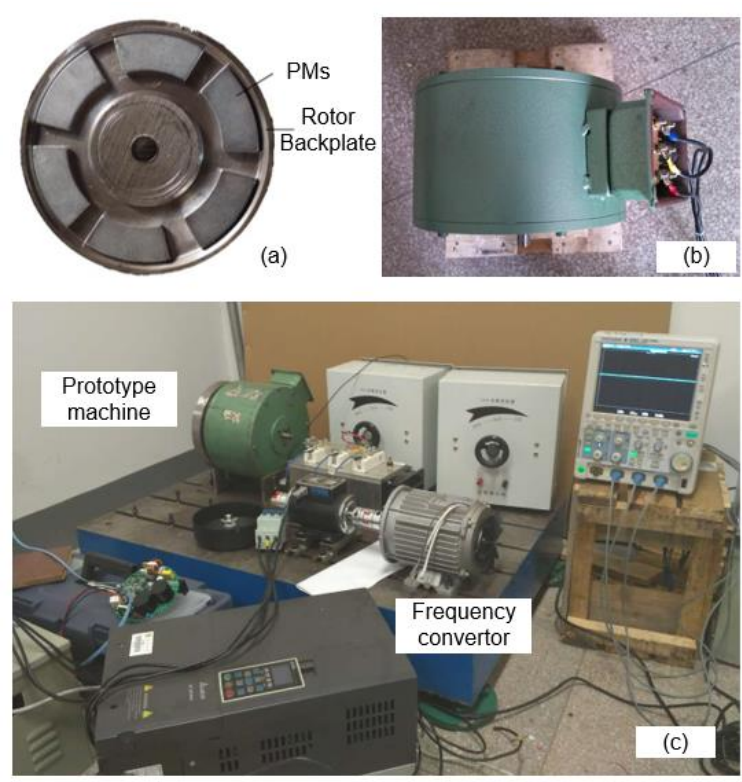

Figure 23. Experimental test rig. 
In terms of computation time, the analytical model requires only 206 seconds to obtain the cogging torque and electromagnetic torque with one mechanical cycle for eccentricities condition. The 3D FE model, on the other hand, requires almost 27 hours to compute the eccentric performance for one electrical cycle. Although the analysis shows a small error compared with the experiments, it is still acceptable and thus, it can be regarded as a meaningful approach which could save time and achieve an acceptable result. Moreover, the analytical model could be used for further calculation, e.g., optimization.

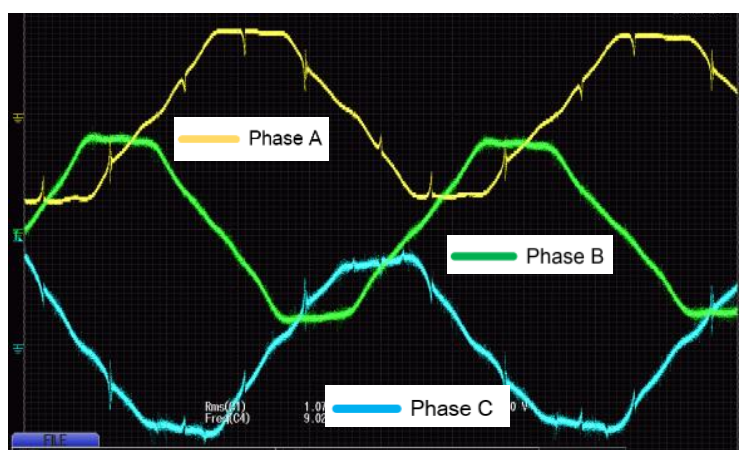

(a)

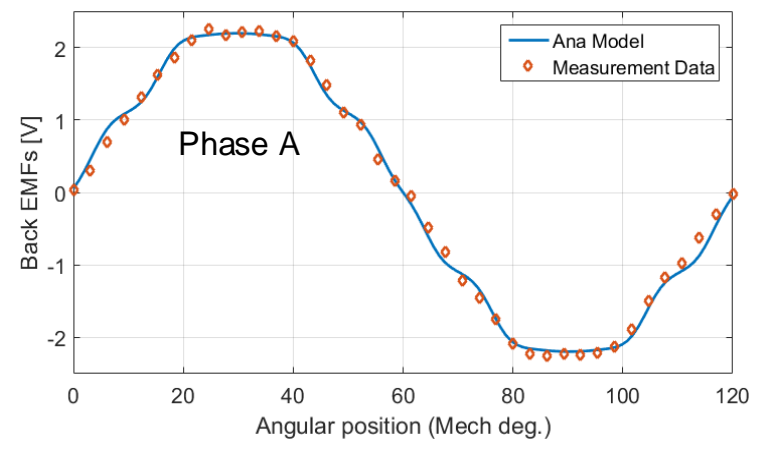

(b)

Figure 24. Experimental Back EMF waveform of AFPMMs (a) and the comparison with analytical model (b).

Table 2. Comparison of Back EMF with Experiment Data.

\begin{tabular}{cccc}
\hline & FE model & Analytical model & Experiment \\
\hline Back EMF Coefficient & 5.30 & 5.30 & 5.28 \\
Error in Back-EMF & $0.37 \%$ & $0.37 \%$ & \\
\hline
\end{tabular}

\section{Conclusion}

In this paper an approach has been presented for modeling double rotor AFPMM with different types of eccentricity. To account for the 3D intrinsic nature of AFPM machine, quasi-3D method is used. The magnetic field, cogging torque and output torque of AFPM machine under healthy and various eccentricities are calculated and the accuracy of the method is verified using 3D FE model. In addition, it can be found that the eccentricity has little effect on the back EMF for static/dynamic angular eccentricities. However, the axis eccentricity conditions could be detected by analyzing the amplitude and phase of back EMFs

Also, this paper has a contribution on the computation time that can be reduced remarkably while maintaining the high accuracy as the FE model. Finally, an experiment validation has been carried out to show the accuracy of the proposed method.

Acknowledgments: This work is supported in part by the National Nature Science Foundation of China (Project Number 51377019), the Scientific and Technological Innovation Program of Jiangsu Province (Project Number BY2014127-15) and the Qing Lan Project.

Author Contributions: Each of the authors contributes on performing experiments and writing articles.

Conflicts of Interest: The authors declare no conflict of interest.

\section{References}

1. Chun, Yon-Do, Dae-Hyun Koo, and Yun-Hyun Cho. "Multiobjective Optimization Design of Axial Flux Permanent Magnet Motor." International Journal of Applied Electromagnetics and Mechanics 25, no. 1-4 (2007): 613-619. 
2. Cvetkovski, Goga, and Lidija Petkovska. “Multi-Objective Approach of Design Optimisation of Axial Flux Permanent Magnet Motor." Edited by Paolo Di Barba and Stawomir Wiak. International Journal of Applied Electromagnetics and Mechanics 51, no. s1 (April 7, 2016): S115-23. doi:10.3233/JAE-2006.

3. W. Hua and L. K. Zhou, "Investigation of a Co-Axial Dual-Mechanical Ports Flux-Switching Permanent Magnet Machine for Hybrid Electric Vehicles," Energies, vol. 8, no. 12, pp. 14361-14379, Dec. 2015.

4. Yao, Hongyang, Hongmei Li, and Yanan Zhou. "Design and Optimization of a Surface Mounted Transverse Flux Permanent Magnet Machine." International Journal of Applied Electromagnetics and Mechanics 51, no. 1 (April 13, 2016): 37-49. doi:10.3233/JAE-150159.

5. T. D. Nguyen, K.-J. Tseng, S. Zhang, and T. D. Nguyen, “A Novel Axial Flux Permanent-Magnet Machine for Flywheel Energy Storage System: Design and Analysis," IEEE Trans. Ind. Electron., vol. 58, no. 9, pp. 3784-3794, Sep. 2011.

6. D. N. Mbidi, K. Van der Westhuizen, R. Wang, M. J. Kamper, and J. Blom, “Mechanical design considerations of a double stage axial-flux PM machine," in Conference Record of the 2000 IEEE Industry Applications Conference, 2000, 2000, vol. 1, pp. 198-201 vol.1.

7. O. O. Ogidi, P. S. Barendse, and M. A. Khan, “Detection of Static Eccentricities in Axial-Flux PermanentMagnet Machines With Concentrated Windings Using Vibration Analysis," IEEE Trans. Ind. Appl., vol. 51, no. 6, pp. 4425-4434, Nov. 2015.

8. M. Thiele and G. Heins, “Computationally Efficient Method for Identifying Manufacturing Induced Rotor and Stator Misalignment in Permanent Magnet Brushless Machines.," IEEE Trans. Ind. Appl., vol. PP, no. 99, pp. 1-1, 2016.

9. A. Di Gerlando, G. M. Foglia, M. F. Iacchetti, and R. Perini, "Effects of Manufacturing Imperfections in Concentrated Coil Axial Flux PM Machines: Evaluation and Tests," IEEE Trans. Ind. Electron., vol. 61, no. 9, pp. 5012-5024, Sep. 2014.

10. S. M. Mirimani, A. Vahedi, and F. Marignetti, "Effect of Inclined Static Eccentricity Fault in Single StatorSingle Rotor Axial Flux Permanent Magnet Machines," IEEE Trans. Magn., vol. 48, no. 1, pp. 143-149, Jan. 2012.

11. J. Li, R. Qu, and Y.-H. Cho, "Effect of unbalanced and inclined air-gap in double-stator inner-rotor axial flux permanent magnet machine," in 2014 International Conference on Electrical Machines (ICEM), 2014, pp. 502-508.

12. J. T. Li, Z. J. Liu, and L. H. A. Nay, "Effect of Radial Magnetic Forces in Permanent Magnet Motors With Rotor Eccentricity," IEEE Trans. Magn., vol. 43, no. 6, pp. 2525-2527, Jun. 2007.

13. A. Bellini, F. Filippetti, C. Tassoni, and G. A. Capolino, "Advances in Diagnostic Techniques for Induction Machines," IEEE Trans. Ind. Electron., vol. 55, no. 12, pp. 4109-4126, Dec. 2008.

14. S. M. Mirimani, A. Vahedi, F. Marignetti, and R. Di Stefano, "An Online Method for Static Eccentricity Fault Detection in Axial Flux Machines," IEEE Trans. Ind. Electron., vol. 62, no. 3, pp. 1931-1942, Mar. 2015.

15. K. Abbaszadeh and S. S. Maroufian, "Axial flux permanent magnet motor modeling using magnetic equivalent circuit," in 2013 21st Iranian Conference on Electrical Engineering (ICEE), 2013, pp. 1-6.

16. S. M. Mirimani, A. Vahedi, F. Marignetti, and E. De Santis, "Static Eccentricity Fault Detection in SingleStator -Single-Rotor Axial-Flux Permanent-Magnet Machines," IEEE Trans. Ind. Appl., vol. 48, no. 6, pp. 1838-1845, Nov. 2012.

17. E. Ajily, M. Ardebili, and K. Abbaszadeh, "Magnet Defect and Rotor Eccentricity Modeling in Axial-Flux Permanent-Magnet Machines via 3-D Field Reconstruction Method," IEEE Trans. Energy Convers., vol. PP, no. 99, pp. 1-10, 2015.

18. T. Mark, "Aanlysis of cogging torque due to manufacturing variations in fractional pitch permanent magnet synchronous machines," doctoral, Charles Darwin University, 2013.

19. E. Ajily, K. Abbaszadeh, and M. Ardebili, “Three-Dimensional Field Reconstruction Method for Modeling Axial Flux Permanent Magnet Machines," IEEE Trans. Energy Convers., vol. 30, no. 1, pp. 199-207, Mar. 2015.

20. A. Hemeida and P. Sergeant, "Analytical Modeling of Surface PMSM Using a Combined Solution of Maxwell's Equations and Magnetic Equivalent Circuit," IEEE Trans. Magn., vol. 50, no. 12, pp. 1-13, Dec. 2014.

21. K. Abbaszadeh and A. Rahimi, "Analytical quasi 3D modeling of an axial flux PM motor with static eccentricity fault," Sci. Iran. Trans. Comput. Sci. Eng. Electr., vol. 22, no. 6, p. 2482, 2015. 
22. F. Marignetti, A. Vahedi, and S. M. Mirimani, "An Analytical Approach to Eccentricity in Axial Flux Permanent Magnet Synchronous Generators for Wind Turbines," Electr. Power Compon. Syst., vol. 43, no. 8-10, pp. 1039-1250, Jun. 2015.

23. D. Zarko, D. Ban, and T. A. Lipo, "Analytical calculation of magnetic field distribution in the slotted air gap of a surface permanent-magnet motor using complex relative air-gap permeance," IEEE Trans. Magn., vol. 42, no. 7, pp. 1828-1837, Jul. 2006.

24. D. Žarko, "A systematic approach to optimized design of permanent magnet motors with reduced torque pulsations," Department of Electrical and Computer Engineering, University of Wisconsin-Madison, 2004.

25. E. Ilhan, E. T. Motoasca, J. J. Paulides, and E. A. Lomonova, “Conformal mapping: Schwarz-Christoffel method for flux-switching PM machines," Math. Sci., vol. 6, no. 1, pp. 1-9, Sep. 2012.

26. Y. G. Guo and J. G. Zhu, "Applications of soft magnetic composite materials in electrical machines," Aust. J. Electr. Electron. Eng., vol. 3, no. 1, pp. 37-46, Jan. 2006.

27. O. Maloberti, R. Figueredo, C. Marchand, Y. Choua, D. Condamin, L. Kobylanski, and E. Bommé, “3-D-2D Dynamic Magnetic Modeling of an Axial Flux Permanent Magnet Motor With Soft Magnetic Composites for Hybrid Electric Vehicles," IEEE Trans. Magn., vol. 50, no. 6, pp. 1-11, Jun. 2014.

28. A. Alipour and M. Moallem, "Analytical magnetic field analysis of axial flux permanent-magnet machines using Schwarz-Christoffel transformation," in Electric Machines Drives Conference (IEMDC), 2013 IEEE International, 2013, pp. 670-677.

29. T. A. Driscoll and L. N. Trefethen, Schwarz-Christoffel mapping. Cambridge; New York: Cambridge University Press, 2002.

30. T. C. O'Connell and P. T. Krein, “A Schwarz-Christoffel-Based Analytical Method for Electric Machine Field Analysis," IEEE Trans. Energy Convers., vol. 24, no. 3, pp. 565-577, Sep. 2009. 